\title{
REAL TIME OPTIMIZED TRAFFIC MANAGEMENT ALGORITHM
}

\author{
Partha Sarathi Chakraborty, Prajeeth Nair, Pranshu Raj Sinha and Ishan Kumar \\ Behera
}

Department of Computer Science and Engineering, SRM University, India

\begin{abstract}
In the present scenario, research conducted is mostly based on determining the duration of green light. Moreover the research papers published on Adaptive Traffic Management did not focus much on the concept of handling Emergency Vehicles. This major role of this project is as a continuation to the existing research papers published on this topic. Here we not only handle traffic effectively but also elaborate on effective management of highly prioritized vehicles through all possible phases. In this particular research paper, Wireless Sensor Networks (WSN) is assumed to be the source of input.
\end{abstract}

\section{KEYWORDS}

Wireless Sensor Networks, WSN, Traffic Management.

\section{INTRODUCTION}

The number of vehicles seen has been increasing rapidly over the past few years. The reason for this phenomenon could be attributed to growing population and everyone's desire to possess their own personal mode of transport. To bear this burden is where Intelligent Transportation System's comes into play. ITS or Intelligent Transportation System, as name suggests, provides an effective and intelligent traffic management solutions with a goal to minimize delay in traffic, improvement in traffic, and efficient flow of traffic. Such goal could be achieved through surveillance units like sensors (wired or wireless), Bluetooth, infrared and so on.

The communication system manages the communication between surveillance system and traffic light control system. The traffic light control system implements the dynamic traffic signal control algorithm and displays the corresponding traffic lights (red/green) to various lanes.

\subsection{Responsibility of WSN}

In order to achieve the desired objective the source of input we use is the Wireless Sensor Networks (WSN) which effectively monitors traffic.

\subsection{Need for Dynamic Traffic Signal Control}

There are two main methods in traffic signal control:

i. Periodical signal control.

ii. Dynamic signal control. 
The periodic signal control method uses a pre-defined sequence and duration of green light for various lanes. As name suggests, the first method is ineffective for real time traffic scenarios and therefore has limited efficiency. The dynamic traffic signal control method adapts the traffic signal in accordance with the dynamic traffic flow and thus works effectively for less as well as busy intersections also.

\subsection{Steps of proposed Dynamic Traffic Signal Control Algorithm}

The proposed algorithm contains four steps:

i. $\quad$ Determining queue length (volume) of traffic.

ii. Determining the presence of Emergency Vehicles as well as the scenario (best to worst case)

iii. Assigning green light to most suitable phase.

iv. Calculating green light duration for each phase.

\section{LITERATURE SURVEY}

One of the very first phases of our project was to collect all possible research papers written in any of the National or International Journals for the concerned topic.

These research papers were thoroughly studied and any scope for further improvements was pondered upon. Our project uses these two papers as its base:-

\subsection{Literature Survey [1]}

Title- Dynamic Traffic Signal Control Algorithm in Intelligent Transportation System through Wireless Sensor Networks.

Author- Monica Johri ,Anurag Goel, Ashutosh Kumar Tiwari.

Journal/Conference- International Journal of Engineering \& Science Research

\section{Description -}

i. The flow of traffic through the intersections decides the signal phase selection. The four directions are marked as North, South, East and West, each direction having three lanes, which are turn-left (L), go-forward (F) and turn-right $(\mathrm{R})$.

ii. To formulate the problem, the paper assumes that the left-turn (L) is permitted all the time. So each passing vehicle can have a direction $d$ of $\{\mathrm{E}, \mathrm{S}, \mathrm{N}, \mathrm{W}\}$ and lane 1 of $\{\mathrm{F}, \mathrm{R}\}$.For the emergency vehicles, the proposed algorithm needs some prior information about them such as their position, speed, direction of travelling etc. all of which it receives from the GPS.

All the possible twelve phases are introduced in their proposed algorithm. 


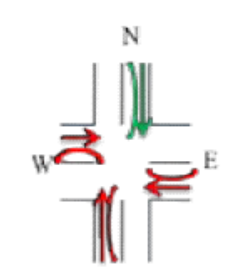

$\mathrm{S}$

Case 1

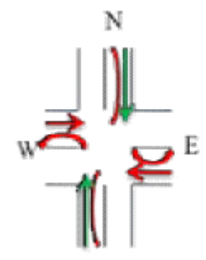

$\mathrm{S}$

Case 5

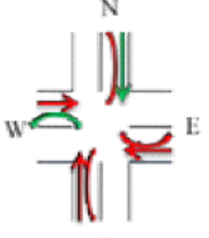

$\mathrm{S}$

Case 9

RIGHT TURN GO

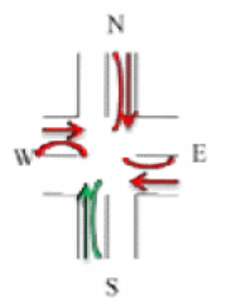

Case 2

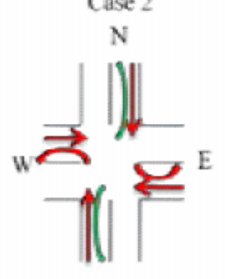

S

Carse 6

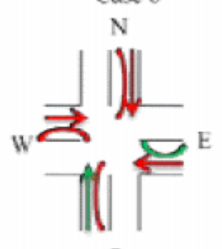

$\mathrm{S}$

Case 10

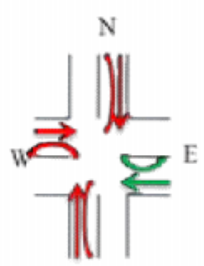

$\mathrm{s}$

Case 3

$\mathrm{N}$

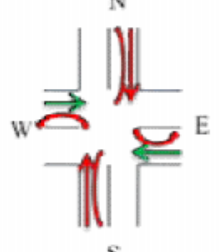

$\mathrm{S}$

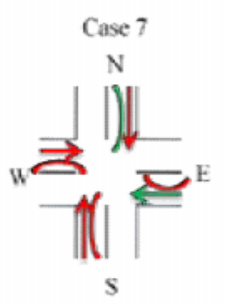

Case 11

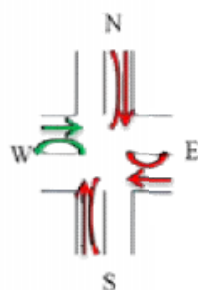

Case 4

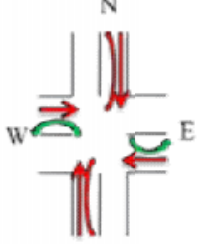

$\mathrm{s}$

Case 8

$\mathrm{N}$

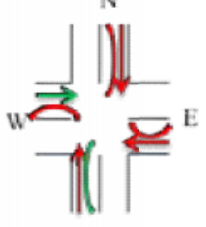

$\mathrm{S}$

Case 12

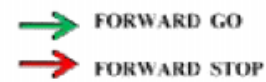

Figure 2.1 The twelve phases

This algorithm considers a number of traffic factors such as Lane waiting queue, Queue passing time, Phase waiting queue, waiting time etc.

\subsection{Base Research Paper [2]}

Title -Intelligent Traffic Light Flow Control SystemUsing Wireless Sensors Networks.

Author -Khalil M. Yousef, Jamal N. Al - Karaki and Ali M. Shatnawi.

Journal/Conference- Journal of Information Science and Engineering 26, 753-768 (2010).

i. A running vehicle can be given as $\{P, D\}$ which results in twelve cases $\{N R, N F, N L$, SR, SF, SL, ER, EF, EL, WR, WF, WL\} where P represents the path and D represents the direction.

ii. In this paper, an intelligent traffic light control system is presented which consists of two parts:

a. Wireless Sensor Network (WSN).

b. Traffic control box (TCB).

WSN is nothing but a network of small nodes known as Traffic Sensor Nodes which gathers information regarding the traffic and stores it in the Base Station.

iii. In this paper, two algorithms are proposed -

i. Traffic system communication algorithm (TSCA).

ii. Traffic signal time manipulation algorithm (TSTMA). 


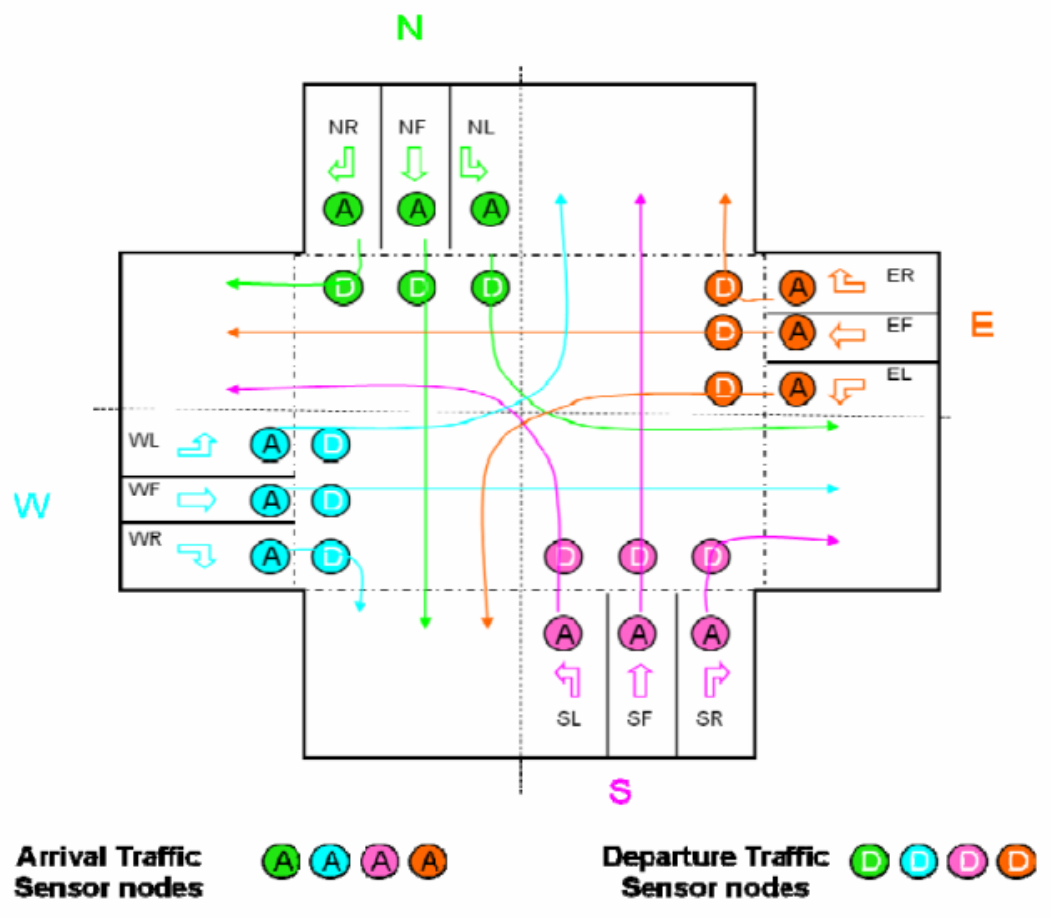

Figure 2.2 Model of Intersection and TSN Architecture

The TSCA controls the communication routes between all the TSNs and BS as well as interfacing with TCB in a simple and efficient manner.

The TSTMA generates a traffic phase plan which is a sequence of four phases and in each phase, two lanes are selected to pass the intersection, on the basis of dynamic traffic volume.

\section{PROBLEM DESCRIPTION}

\subsection{Existing Problem}

Need for detailed approach towards handling prioritized vehicles (also known as emergency vehicles) like Fire Truck, Ambulance and Police Vans by taking into consideration all possible scenarios from best case to worst case.

Reference paper [1] proposes a very basic approach towards handling these vehicles by simply assigning green light for the direction where the emergency vehicle is present. Reference paper [2] however does not consider emergency vehicles at all.

\subsection{Proposed Technique}

i. In our proposed algorithm we offer a solution to tackle the worst case (deadlock) condition for management of emergency vehicles.

This is done as a 2 stage process:

Stage 1 : The first stage categorizes Emergency Vehicles on the basis of Standard policies which may vary from one country to another. This assigns a priority value for each type of Emergency Vehicle. 
Stage 2 : Considering the distance of each of the Emergency vehicle from the intersection point.

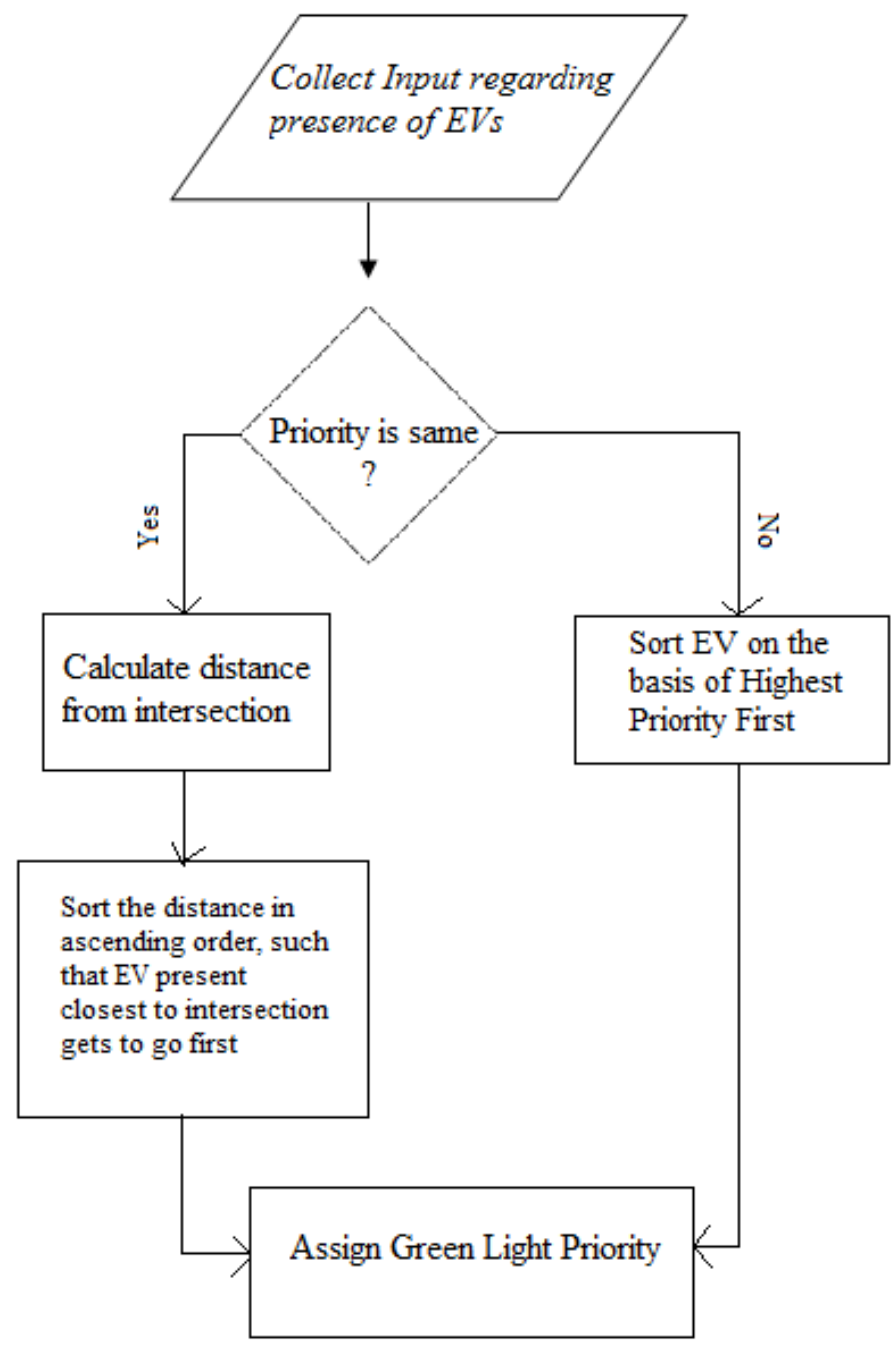

Figure 3.1 Process Flow Chart

ii. As an addition we have also included a totally abstract approach for more Optimized Emergency Vehicle Management by collaborating Code Messaging Service (CMS) and a GPS Enabled Mobile Device. Being an abstract thought, this model serves the purpose of base for possible future research.

\section{PROPOSED METHODOLOGY}

\subsection{Problem Formulation and Assumptions}

\subsubsection{Problem Formulation}

i. The flow of traffic through the intersections decides the signal phase selection.

ii. To formulate a solution, we assume that the left-turn (L) is permitted all the time. 
International Journal of Computer Science \& Information Technology (IJCSIT) Vol 6, No 4, August 2014

iii. All 8 pairs of $(d, 1)$ combine in various combinations which results in twelve possible cases, as shown in figure 4.1 .

\subsubsection{Assumptions}

i. Constant speed of all vehicles of similar type.

ii. It is assumed that all the vehicles have a vehicle node installed in them.

iii. The data regarding traffic volume approaching towards the intersection point from various lanes, which is required for the implementation of proposed dynamic traffic signal control algorithm, is assumed to be getting from the Wireless Sensor Network. The working of WSN is not a part of our paper.
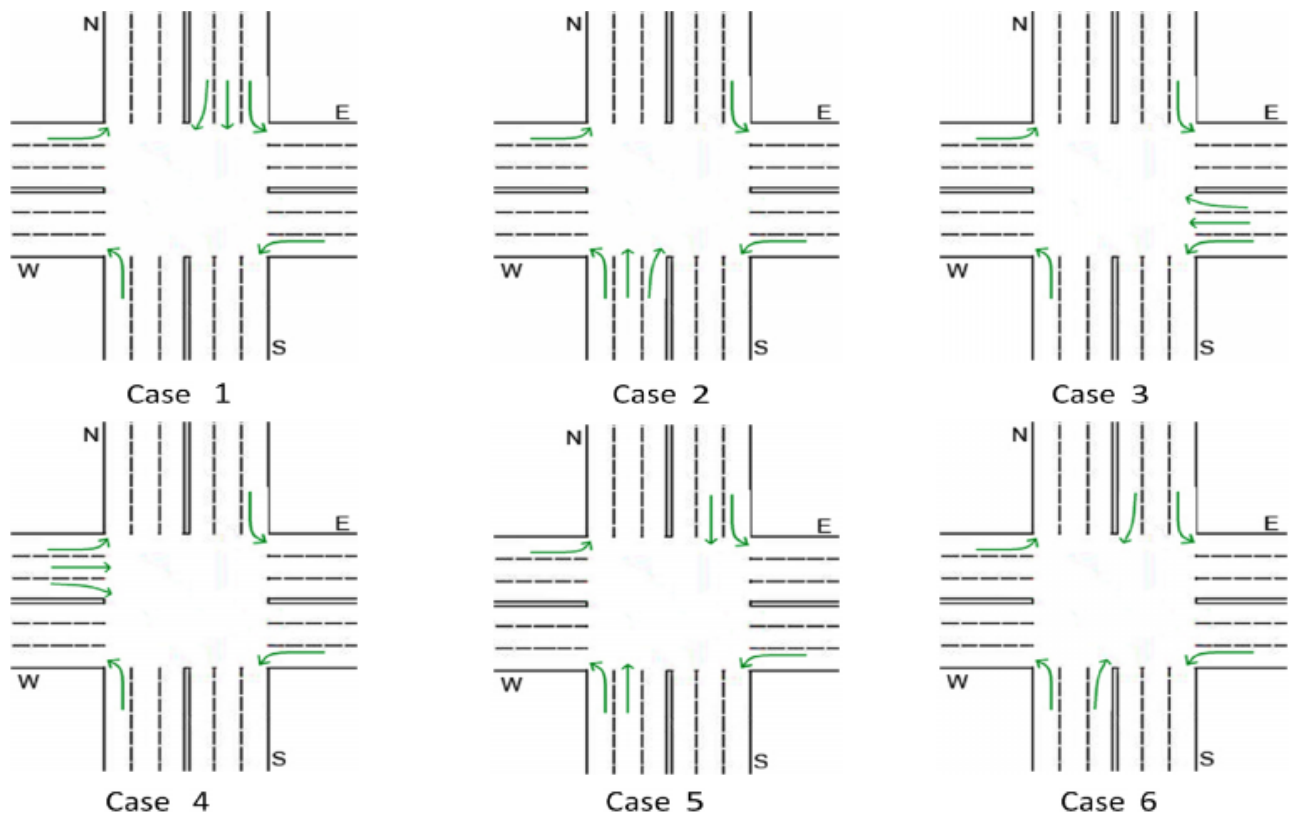


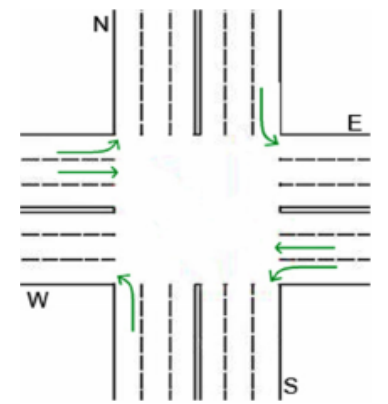

Case 7

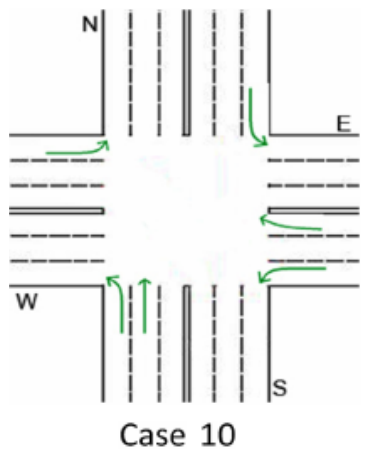

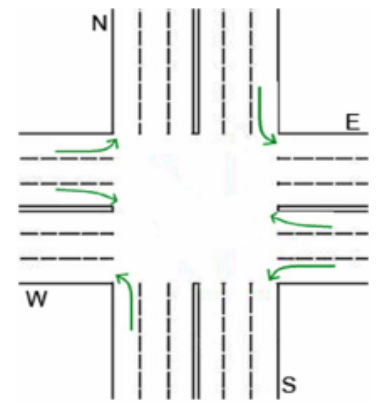

Case 8

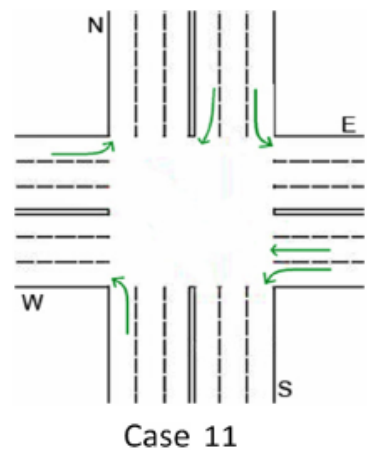

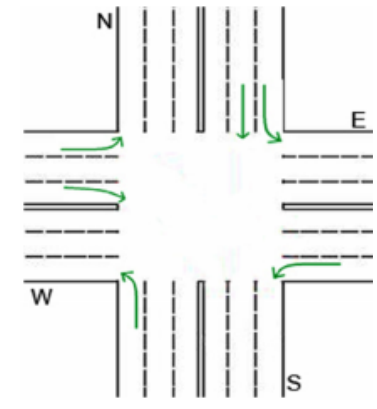

Case 9

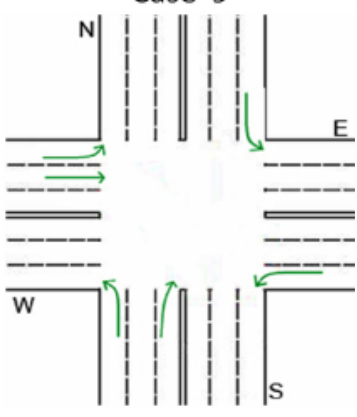

Case 12

Figure 4.1. All possible phases for a 3 Lane intersection

\subsection{Different Problem Notations Used}

The notations depicting directions, lanes and cases are given as:

$\mathrm{D}=\{$ North, South, East, West $\}$.

$\mathrm{L}=\{$ Forward, Right $\}$.

$\mathrm{C}=\{1,2,3,4 \ldots 12\}$.

Here, D, L and C denote the set of directions, lanes and cases respectively.

\subsubsection{Expectant Phase}

Expectant Phase, denoted as $\mathbf{E} \phi$, of a vehicle is defined as the phase in which a vehicle pass the intersection.

Case 01: $\mathrm{E} \phi(\mathrm{N}, \mathrm{F})=\mathrm{E} \phi(\mathrm{N}, \mathrm{R})=\phi \mathrm{a}$.

Case 02: $\mathrm{E} \phi(\mathrm{S}, \mathrm{F})=\mathrm{E} \phi(\mathrm{S}, \mathrm{R})=\phi \mathrm{b}$.

Case 03: $\mathrm{E} \phi(\mathrm{E}, \mathrm{F})=\mathrm{E} \phi(\mathrm{E}, \mathrm{R})=\phi c$.

Case 04: $\mathrm{E} \phi(\mathrm{W}, \mathrm{F})=\mathrm{E} \phi(\mathrm{W}, \mathrm{R})=\phi \mathrm{d}$.

Case 05: $\mathrm{E} \phi(\mathrm{N}, \mathrm{F})=\mathrm{E} \phi(\mathrm{S}, \mathrm{F})=\phi \mathrm{e}$.

Case 06: $\mathrm{E} \phi(\mathrm{N}, \mathrm{R})=\mathrm{E} \phi(\mathrm{S}, \mathrm{R})=\phi \mathrm{f}$.

Case 07: $\mathrm{E} \phi(\mathrm{E}, \mathrm{F})=\mathrm{E} \phi(\mathrm{W}, \mathrm{F})=\phi \mathrm{g}$.

Case 08: $\mathrm{E} \phi(\mathrm{E}, \mathrm{R})=\mathrm{E} \phi(\mathrm{W}, \mathrm{R})=\phi \mathrm{h}$.

Case 09: $\mathrm{E} \phi(\mathrm{N}, \mathrm{F})=\mathrm{E} \phi(\mathrm{W}, \mathrm{R})=\phi \mathrm{i}$.

Case 10: $\mathrm{E} \phi(\mathrm{S}, \mathrm{F})=\mathrm{E} \phi(\mathrm{E}, \mathrm{R})=\phi \mathrm{j}$.

Case 11: $\mathrm{E} \phi(\mathrm{N}, \mathrm{R})=\mathrm{E} \phi(\mathrm{E}, \mathrm{F})=\phi \mathrm{k}$.

Case 12: $\mathrm{E} \phi(\mathrm{S}, \mathrm{R})=\mathrm{E} \phi(\mathrm{W}, \mathrm{F})=\phi 1$. 


\subsubsection{Lane Waiting Queue}

Lane Waiting Queue, denoted as $\mathbf{Q}(\mathbf{d}, \mathbf{l})$, is defined as the number of vehicle waiting on the path $P=\{d, 1\}, d \in D$ and $1 \in L$.

\subsubsection{Phase waiting Queue}

Phase Waiting Queue, denoted as $\mathbf{Q}_{\mathbf{x}}$, is the length of queue for phase $\mathrm{x}$

$$
\mathrm{Q}_{\mathrm{x}}=\operatorname{MAX}(\mathrm{TQ}(\mathrm{d}, 1)) \text {, }
$$

where $(\mathrm{d}, \mathrm{l}) \in\{\mathrm{E} \phi(\mathrm{d}, \mathrm{l})=\phi \mathrm{x}\}$

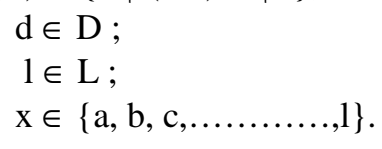

\subsubsection{Queue passing Time}

Queue passing time, represented as TQ $(\mathrm{d}, 1)$, is the time taken by all waiting vehicles to pass the intersection.

$$
\mathrm{TQ}(\mathrm{d}, 1)=\mathrm{T} 1+(\tau *(\mathrm{Q}(\mathrm{d}, 1)-1))
$$

$\mathrm{T} 1$ is the time taken by first vehicle to cross the intersection.

$\tau$ is the time taken by a vehicle to move to the place of front vehicle.

Phase Queue passing time, denoted as $\mathbf{T} \mathbf{Q}_{\mathbf{x}}$, is calculated as -

$$
\begin{aligned}
\mathrm{TQ}_{\mathrm{x}}= & \operatorname{MAX}(\mathrm{TQ}(\mathrm{d}, 1)) \\
\text { where }(\mathrm{d}, \mathrm{l}) & \in\{\mathrm{E} \phi(\mathrm{d}, \mathrm{l})=\phi \mathrm{x}\} \\
\mathrm{d} & \in \mathrm{D} ; \\
1 & \in \mathrm{L} ; \\
\mathrm{x} & \in\{\mathrm{a}, \mathrm{b}, \mathrm{c}, \ldots \ldots \ldots . ., 1\} .
\end{aligned}
$$

\subsubsection{Emergency condition} vehicle.

Emergency condition, EC (d, l), determines the presence of any kind of Emergency

\subsubsection{Waiting Time}

Waiting Time, WT (d, l), gives the waiting time for the vehicle in front.

\subsubsection{Threshold Waiting Time}

Threshold waiting time, $\mathrm{T}_{\text {threshold }}$, sets the maximum waiting time.

\subsubsection{Phase Time}

Phase time, $\mathrm{TP}_{\mathrm{x}}$, defines duration of green light for phase $\mathrm{x}$

$$
\mathrm{TP}_{\mathrm{x}}=\mathrm{MIN}\left(\mathrm{TQ}_{\mathrm{x}}, \mathrm{TP}_{\max }\right)
$$

\subsubsection{Maximum Phase Time} is allotted.

Maximum phase time, denoted as $\mathrm{TP}_{\max }$, gives the maximum time for which green light

\subsection{The main steps in Dynamic Traffic Signal Control Algorithm}

The proposed dynamic traffic signal control algorithm contains mainly four steps:

i. Determining the queue length (volume) of traffic.

ii. Check for the presence of Emergency Vehicles as well as deciding the current scenario (best or worst). 
iii. Assigning green light to the most suitable phase.

iv. Calculating how much time must be allotted to the phase decided in (iii).

\subsubsection{Traffic Volume Detection}

In the first step of our algorithm the queue length $\left(Q_{x}\right)$ for each phase is computed. This gives us a fair idea regarding the number of vehicles present in each phase. The source of input for this stage of our algorithm is WSN.

\subsubsection{Check for Emergency Vehicle Presence}

In the second step of the algorithm we make a routine check in order to ensure whether an Emergency vehicle is present at the intersection or not.

An Emergency vehicle is a vehicle which has to be assigned a greater priority than other motorized vehicles.

For this purpose we first need to assign a priority for different types of emergency vehicles. For example Fire Trucks can be assigned priority 0(highest) followed by ambulance and so on. Assigning priority for the Emergency Vehicles is totally dependent on the standard policies, taking into consideration multiple parameters, and may vary from country to country.

Considering all probable scenarios, we classify the management of Emergency Vehicles on the basis of 4 cases:

CASE 1 : Only One Emergency Vehicle is Present

In this case we simply assign green light to the lane where the Emergency Vehicle is present.

CASE 2 : $\quad$ Two Emergency Vehicles each having different Priority In this case we simply allow the vehicle with the higher priority to pass through first followed by the vehicle with lower priority.

CASE 3 : Two Emergency Vehicles each having same Priority This is a typical scenario since both the Emergency Vehicles have the same priority. In order to tackle such a situation we need to be fair to both these vehicles. Therefore we assign green light priority first to the Emergency Vehicle present at the shortest distance from the intersection.

CASE 4 : $\quad$ Four Emergency Vehicles (all different priorities) placed at each of the four lanes [DEADLOCK]

Such a situation can be rightly tackled by assigning Green light to the Emergency Vehicle on the basis of Highest Priority First (HFS) Scheduling.

\subsubsection{Assigning Green Light to most suitable phase}

In this step, the queue length $\left(\mathrm{Q}_{\mathrm{x}}\right)$ is calculated as per the input given by the Wireless Sensor Network.

\subsubsection{Determination of Green Light Duration}

The third step is to determine as to how much time green light should be assigned to a phase. The maximum phase time should not be more than 120 seconds. 


\subsection{The Proposed Dynamic Traffic Signal Control Algorithm}

$\overline{\text { Dynamic Traffic Signal Control Algorithm }}$

I/Initialization - Here parameters like Max Time and threshold time is initialized

1. Initialize $\mathrm{T}_{\max }=90, \mathrm{~T}_{\text {threshold }}=120$.

Set $\mathrm{Q}(\mathrm{d}, \mathrm{l}) \forall(\mathrm{d}, \mathrm{l})$, where $\mathrm{d} \in \mathrm{D}, \mathrm{l} \in \mathrm{L}$.

$\mathrm{Q}(\mathrm{d}, 1)$ contains the number of vehicles on the path $\mathrm{P}=\{\mathrm{d}, 1\}$

//Traffic Volume Detection - Computing the queue length for each lanes

2. Compute $\mathrm{Q}_{\mathrm{x}}=\operatorname{MAX}(\mathrm{Q}(\mathrm{d}, 1)) \forall \mathrm{x}$, where $\mathrm{x} \in\{\mathrm{a}, \mathrm{b}, \ldots, 1\}, \mathrm{d} \in \mathrm{D}, 1 \in \mathrm{L}$.

Qx contains the maximum queue length of each of the 12 phases

/*Emergency Check and Traffic Phase Selection - Here check for the presence of emergency vehicles is made so as to assign green light to the most suitable phase */

3. Check emergency condition $\forall \mathrm{d}, \mathrm{d} \in \mathrm{D}$.

4. If direction d'has greater priority then goto 5 else goto 7

5. If(Emergency vehicle only in one direction $\left.\mathrm{d}^{\prime}\right)$

Assign green light to phase $\phi x$ next such that $\left\{\mathrm{E} \phi\left(d^{\prime}, 1_{1}\right)=E \phi\left(d^{\prime}, l_{2}\right)\right\}, 1_{1}, l_{2} \in L$.

$\mathrm{d}^{\prime}=$ direction of emergency vehicle

$1_{1}, l_{2}=$ all the 2 possible lanes

6. Else If (Emergency vehicle in more than one direction but of different priorities)

a) Assign count= number of emergency vehicles in different direction

b) For $k=1$ to count

Loop

Int priority $[\mathrm{k}]=$ Priority of emergency vehicle for each direction

Char $\operatorname{dir}[\mathrm{k}]=$ direction of presence of Emergency Vehicle

End Loop

c) Sort the array priority[] in ascending order of priority and correspondingly change the value of $\operatorname{dir}[]$

d) For I = 1 to count

Loop

Assign green light to $\mathrm{E} \phi\left(\operatorname{dir}[\mathrm{i}], \mathrm{l}_{1}\right)=\mathrm{E} \phi\left(\operatorname{dir}[\mathrm{i}], \mathrm{l}_{2}\right)=\phi \mathrm{x}$

End Loop

7. Else

a) Assign count $=$ number of emergency vehicles

b) For $\mathrm{I}=1$ to count

Loop

Int $\operatorname{arr}[\mathrm{i}]=$ distance from intersection of emergency vehicle for each direction

Char $\operatorname{dir}[\mathrm{i}]=$ direction of presence of Emergency Vehicle

End Loop

c) Sort the array $\operatorname{arr}[]$ in ascending order of distance and correspondingly change the value of $\operatorname{dir}[]$

d) For I = 1 to count

Loop

Assign green light to $\mathrm{E} \phi\left(\operatorname{dir}[\mathrm{i}], 1_{1}\right)=\mathrm{E} \phi\left(\operatorname{dir}[\mathrm{i}], 1_{2}\right)=\phi \mathrm{x}$

End Loop

8. Compute $\mathrm{WT}(\mathrm{d}, \mathrm{l}) \forall(\mathrm{d}, \mathrm{l})$, where $\mathrm{d} \in \mathrm{D}, \quad l \in \mathrm{L}$.

9. If there exists a path $\left(\mathrm{d}^{\prime}, \mathrm{l}^{\prime}\right)$ with $\mathrm{WT}\left(\mathrm{d}^{\prime}, \mathrm{l}^{\prime}\right) \geq \mathrm{T}_{\text {threshold }}$ then

10.Compute $\mathrm{Q}_{\mathrm{x}}=\operatorname{MAX}\left(\mathrm{Q}\left(\mathrm{d}^{\prime}, \mathrm{l}^{\prime}\right), \mathrm{Q}(\mathrm{d}, 1)\right)$, such that $\left\{\mathrm{E} \phi\left(\mathrm{d}^{\prime}, \mathrm{l}^{\prime}\right)=\mathrm{E} \phi(\mathrm{d}, \mathrm{l})=\phi \mathrm{x}\right\}$,

for every possible $\mathrm{x}, \mathrm{x} \in\{\mathrm{a}, \mathrm{b}, \mathrm{c}, \ldots, 1\}, \mathrm{d} \in \mathrm{D} ; 1 \in \mathrm{L}$. 
11. Assign green light to phase $\phi x$ having maximum value of $Q_{x}$ computed above, next.

12. Else

Assign green light to phase $\phi x$ having maximum value of $Q_{x}$ computed in step 2, next.

13. End if.

14. End if.

//Determination of Green Light Duration - Calculating the duration of green light

15. Compute $\mathrm{TQ}_{\mathrm{x}}=\mathrm{T}_{1}+\left(\tau *\left(\mathrm{Q}_{\mathrm{x}}-1\right)\right)$.

16. Compute $\mathrm{TP}_{\mathrm{x}}=\mathrm{MIN}\left(\mathrm{TQ}_{\mathrm{x}}, \mathrm{TP}_{\max }\right)$.

17. Set the green light time for phase $\phi x$ for time $\mathrm{TP}_{\mathrm{x}}$.

Note : $Q_{x}$ as well as $T Q_{x}$ are changed dynamically according to the traffic flow.

\section{RESULT}

The efficiency of our solution to the existing shortcoming is expressed on the basis of theoretical and physical factors.

On theoretical basis, our algorithm is a success because we have tried to provide solution to a very new problem of Emergency Deadlock condition, which opens a new dimension for further research and exploration of more complex traffic situations and development of their solutions.

On the basis of physical factors like intersection throughput factor etc. our algorithm is nearly similar to the one presented in our base research paper[1]. But due to the introduction of new concept in the algorithm, there has been some negligible effects on the time complexity.

In computer science, the Time Complexity of an algorithm quantifies the amount of time taken by an algorithm to run as a function of the length of the string representing the input.

For the above proposed algorithm, the entire time complexity relies on the sorting technique used in order to sort the distance of emergency vehicles from the intersection as well as to sort the pre-defined priority. In order to maximize the efficiency, the sorting technique considered is Merge Sort [11] with best case, worst case and average case as $\mathrm{O}$ (nlogn).

Therefore the overall time complexity for the proposed algorithm is $\mathrm{O}$ (nlogn).

\subsection{Comparing Efficiency with Base Algorithm}

Keeping the time complexity as the medium of consideration we compared the time taken by both these algorithms for different values of ' $n$ '.

\begin{tabular}{|r|rr|r|}
\hline Value of N & Base Algorithm & \multicolumn{1}{|c|}{ Proposed Algorithm } \\
\hline 1 & 0 & 1 \\
\hline 2 & 1 & 2 \\
\hline 3 & 2 & 4.5 \\
\hline 4 & 3 & 8 \\
\hline 5 & 4 & 11.5 \\
\hline
\end{tabular}

Table 5.1 Time Complexity for different values 


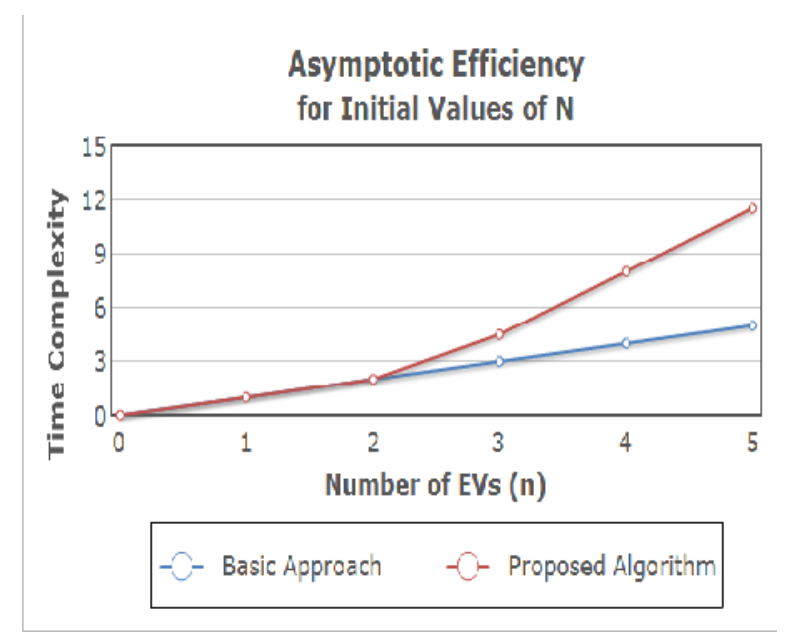

Figure 5.1: Algorithm Efficiency Graph

It is evident from above that with increasing number of emergency vehicles (n) the overall time consumption by our algorithm would increase quite a lot in comparison to the existing (base) algorithm. The results are not surprising since we have been giving a lot of emphasis on priority vehicles, so a considerable deviation of time complexity is expected.

However, throwing some positive light, this deviation may not be taken as concern since even in worst case scenario the number of emergency vehicles present at the intersection would be limited. So there is no need to consider higher values of 'n'.

Remaining graphs generated are in close reference to Base Paper [1] since our objective was to provide a meaningful improvement or an "add-on" to the existing base algorithm. We have successfully accomplished our desired objective, the result of which has been verified after showcasing the technical implementation.

\section{CONCLUSION AND FUTURE WORK}

\subsection{CONCLUSION}

Wireless sensor networks offer a promising platform for traffic monitoring that can compete with current technology in terms of accuracy and lifetime. In this paper, we have given an extension to the existing dynamic traffic signal control algorithm keeping in mind the concrete objective of minimizing the average waiting time. Here we also include a scenario involving the worst case (deadlock) condition for management of emergency vehicles. This algorithm offers a solution to the above mentioned problem which has not yet been approached in any of the published research papers. This project concludes with a concept solution for internal prioritization of ambulances which offers a wide scope for future development.

\subsection{FUTURE WORK}

In our future research, the proposed dynamic traffic signal control algorithm can be extended by eliminating the above made assumptions such the vehicles approaching towards the intersection from all the directions are ideal sized vehicles are assumed to be ideal sized. This assumption 
can be removed by adding the detection of heavy vehicles. Moreover a better and much more feasible solution to the problem of Internal Priority for Ambulances could be proposed in future.

\section{APPENDIX}

\section{A. Why WSN?}

i. Here we observe what all kinds of properties different surveillance units possess:-

\begin{tabular}{|l|l|l|l|l|l|l}
\hline \multicolumn{2}{|l|}{ Technology } & \multicolumn{4}{l}{ Data Type } & \multicolumn{2}{l}{} \\
\hline \multicolumn{2}{|l|l|l|l|l|l|l}{} & Count & Speed & Classification & Occupancy & Presence \\
\hline \multicolumn{2}{|l|}{ Intrusive } & & & & & \\
\hline & Inductive Loop & $\mathrm{Y}$ & $\mathrm{Y}$ & $\mathrm{Y}$ & $\mathrm{Y}$ & $\mathrm{Y}$ \\
\hline & pneumatic road tube & $\mathrm{Y}$ & $\mathrm{Y}$ & $\mathrm{Y}$ & $\mathrm{N}$ & $\mathrm{N}$ \\
\hline & & & & & \\
\hline Non-Intrusive & & & & & \\
\hline Video Image Processing & $\mathrm{Y}$ & $\mathrm{Y}$ & $\mathrm{Y}$ & $\mathrm{Y}$ & $\mathrm{Y}$ \\
\hline & Ultrasonic & $\mathrm{Y}$ & $\mathrm{N}$ & $\mathrm{N}$ & $\mathrm{N}$ & $\mathrm{Y}$ \\
\hline Wireless Sensor Network & & & & & \\
\hline & Magnetometer & $\mathrm{Y}$ & $\mathrm{Y}$ & $\mathrm{Y}$ & $\mathrm{Y}$ & $\mathrm{Y}$ \\
\hline
\end{tabular}

Table A.1 Characteristics of different Surveillance Systems [3]

Y-Available N- Not Available

ii. Inductive loop detector is often considered to be one of the most accurate surveillance system.

\begin{tabular}{|c|c|c|c|c|c|}
\hline \multicolumn{2}{|c|}{ System } & \multirow[t]{2}{*}{ Mounting } & \multicolumn{2}{|c|}{ Error [\%] } & \multirow[t]{2}{*}{ Sources } \\
\hline & & & Count & Speed & \\
\hline \multicolumn{6}{|c|}{ Inductive Loop } \\
\hline & Saw-cut & Pavement & $0.1-3$ & $1.2-3.3$ & MNDOT[2.26] \\
\hline \multicolumn{6}{|c|}{ Pneumatic Road tube } \\
\hline & & Pavement & $0.92-30$ & & SDDOT[2.27] \\
\hline \multicolumn{6}{|c|}{ Video Image Processing } \\
\hline & Autoscope solo & Side-fire & 5 & 8 & MNDOT[2.26] \\
\hline & Autoscope solo & Overhead & 5 & $2.5-7$ & MNDOT[2.26] \\
\hline \multicolumn{6}{|c|}{ Ultrasonic } \\
\hline & Lane King & Overhead & 1.2 & & MNDOT[2.28] \\
\hline \multicolumn{6}{|c|}{ Wireless Sensor Networks } \\
\hline & VSN240 & Pavement & $1-3$ & & [section 4.4] \\
\hline
\end{tabular}

Table A.2 Error Rates for Different Surveillance Systems [3] 
International Journal of Computer Science \& Information Technology (IJCSIT) Vol 6, No 4, August 2014

iii. Environmental factors that affect these traffic surveillance technologies

\begin{tabular}{|c|c|c|c|c|c|}
\hline \multirow[t]{2}{*}{ Technology } & \multicolumn{5}{|c|}{ Environmental Factor } \\
\hline & Penetration & Wind & Temperature & Lighting & $\begin{array}{l}\text { High traffic } \\
\text { flow }\end{array}$ \\
\hline \multicolumn{6}{|l|}{ Intrusive } \\
\hline Inductive Loop & & & $Y$ & & \\
\hline Pneumatic Road Tube & & & Y & & Y \\
\hline \multicolumn{6}{|l|}{ Non-Intrusive } \\
\hline Video Image Processing & $\mathrm{Y}$ & $Y$ & $\mathrm{Y}$ & $Y$ & \\
\hline Ultrasonic & & & & & \\
\hline \multicolumn{6}{|l|}{ Wireless Sensor Network } \\
\hline Magnetometer & & & $Y$ & & \\
\hline
\end{tabular}

Table A.3 Environmental Factors affecting various Surveillance Technologies [3]

iv. Calculating the Life Cycle Cost

Formula Used to calculate Lifecycle Cost:

$\mathrm{LC}=((\text { device cost*quantity })+\text { installation cost }+ \text { maintenance cost })^{*}$

$$
\left\lfloor\frac{(1+i)^{n}-1}{i(1+i)^{n}}\right\rfloor
$$

$\mathrm{I}=4.1$ (considered to be inflation rate)

$\mathrm{n}=$ Life Time in (given as number of years)

\begin{tabular}{|l|l|l|l|l|l|}
\hline Technology & $\begin{array}{l}\text { Device cost* } \\
\text { Quantity }\end{array}$ & $\begin{array}{l}\text { Installation } \\
\text { cost }\end{array}$ & $\begin{array}{l}\text { Maintenance } \\
\text { cost }\end{array}$ & Lifetime $(\mathrm{n})$ & $\begin{array}{l}\text { Lifecycle cost } \\
\text { (in } \$ \text { ) }\end{array}$ \\
\hline $\begin{array}{l}\text { Inductive } \\
\text { loop }\end{array}$ & $12 \times 750=9000$ & $\begin{array}{l}\text { Included in } \\
\text { device cost }\end{array}$ & 700 & 10 & 2365.85 \\
\hline $\begin{array}{l}\text { Saw-cut } \\
\text { Proco Image }\end{array}$ & $2 \times 4900=9800$ & 1000 & 400 & 10 & 2731.70 \\
$\begin{array}{l}\text { Autoscope } \\
\text { solo }\end{array}$ & & & & 10 & 1414.63 \\
\hline $\begin{array}{l}\text { WSN } \\
\text { VSN240 }\end{array}$ & $450 \times 12=5400$ & 200 & 200 & & 10 \\
\hline
\end{tabular}

Table A.4 Life Cycle Cost Calculation [3]

The values calculated as the life cycle cost does not include the traffic delay cost caused by disrupting the traffic during future maintenance. Overall the Wireless Sensor Network has proved to be better than compared to other traffic surveillance technologies. 
International Journal of Computer Science \& Information Technology (IJCSIT) Vol 6, No 4, August 2014

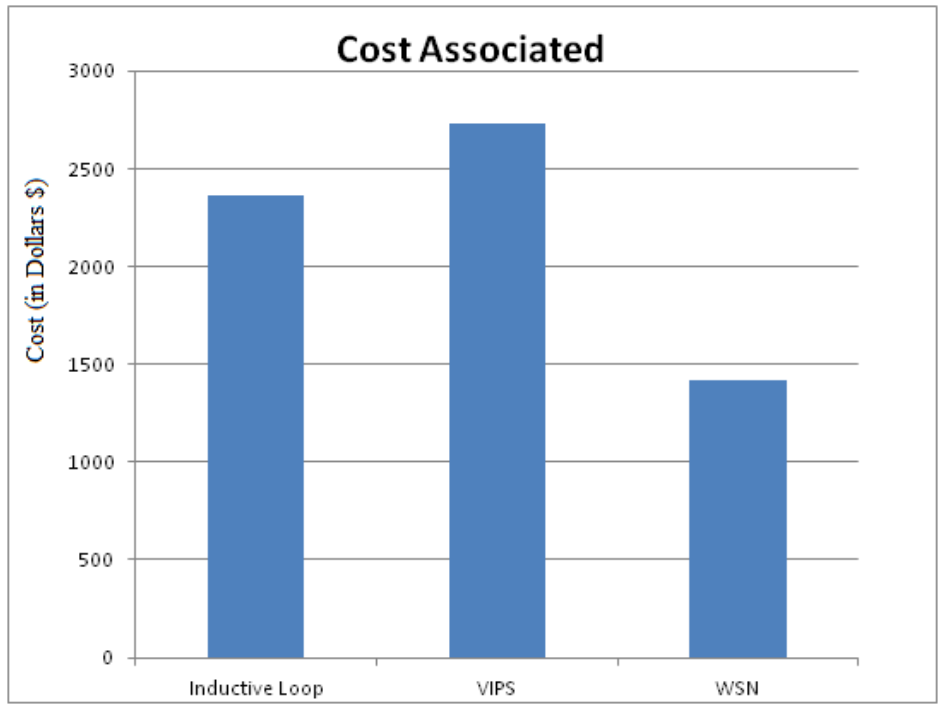

\section{B. Network Architecture}

The proposed sensor layout is given as:-

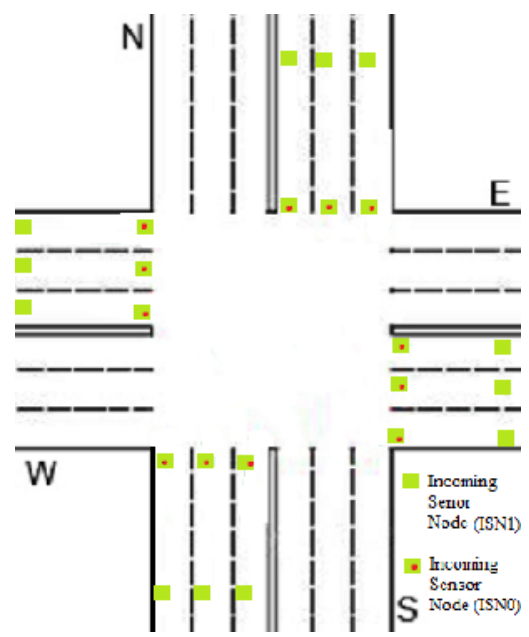

Figure B1: Sensor Layout

There are 3 Incoming Sensor Nodes (ISN) placed on each of the incoming lanes ISN-0 are the sensors placed closest to intersection

ISN-1 are the sensors placed at a particular distance from the intersection

Overall there are 24 sensors placed along the intersection.

\section{Three Layered Network Architecture}

To implement the designed algorithm we assume a Three Layer WSN Architecture (W3 Architecture). 
Here we introduce a sensor like node to be installed at each and every vehicle. The primary objective of introducing W3 Architecture is to solve the problem of vehicle identification that can be implemented in a variety of applications like vehicle speed detection, tracking in case of thefts etc.

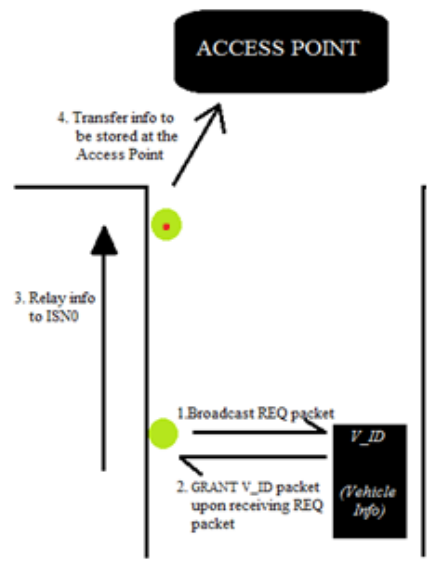

Figure B2 : 3 Layer Architecture

The three layers are given as:
a) LAYER 1
ISNO $\rightarrow$ Access Point
b) LAYER $2 \quad: \quad$ ISN1 $\rightarrow$ ISNO
c) LAYER 3 : $\quad$ Vehicle Node $\rightarrow$ ISN1

a) Just like a handshaking signal in network communication, ISN1 broadcasts REQ (Request) packet. Upon receiving the signal the vehicle node which is installed inside the vehicle grants its identification (V_ID) packet which contains information regarding its Id number, speed, type etc.

b) The sensor node ISN1 then relays the information to its successor, ISNO.

c) ISN0 forwards the information to the Access Point where it is stored in the database using the vehicle id as the primary key in order to avoid repetition of vehicles. The Access point is the Base Controller where our algorithm will run, by using information stored in the database.

A vehicle node is usually in a sleeping state. When a vehicle comes into the road where sensor nodes are installed, vehicle node switches to the active state [6].

\section{C: INTERNAL PRIORITY TO EMERGENCY VEHICLES}

\section{Measuring the Level of Emergency (For Ambulances)}

There is a need to assign a first degree priority to emergency vehicles, most notably; an ambulance on the basis how critical the patient is. This is needed since a person suffering from a critical illness may need to reach the hospital more quickly than a person whose condition is less severe. 
We begin with certain assumptions as follows:-

i. First of all, we begin with the assumption regarding the passengers in the ambulance vehicle.
a) Driver : $\quad$ The person who drives the ambulance
b) Patient : $\quad$ The patient who may/may not be critically ill.
c) Nurse : $\quad$ The nurse acts as knowledgeable person who can make the most accurate judgment regarding the condition of the patient. He / She is needed since a doctor cannot make appropriate decision over the phone.

d) Family Members (optional).

ii. The nurse inside the ambulance is equipped with a standardized mobile device. This mobile device has functionalities of GPS (Global Positioning System) and Code Messaging Service (CMS).

\section{HOW IT WORKS}

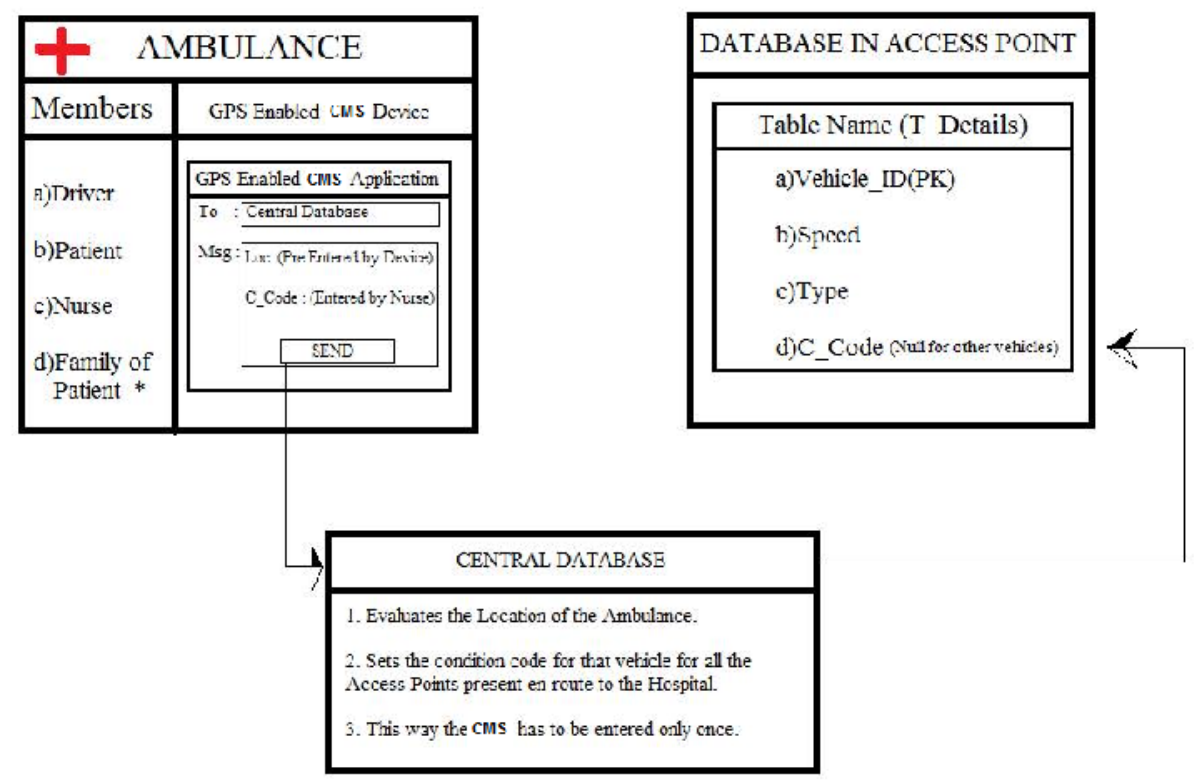

Figure C1: A Proposed Solution for Internal Priority to Emergency Vehicles

iii. Initially we need a set of condition code for all possible conditions the patient may suffer from.

iv. These condition codes are nothing but a set of integers (starting from 0 ) which depicts the severity of the patient.

v. Every ambulance must have a chart depicting the condition code along with its description. 
For example

\begin{tabular}{|c|c|}
\hline \multicolumn{2}{|c|}{ POSSIBLE MEDICAL CHART } \\
\hline Condition Code & Description \\
\hline 0 & $\begin{array}{l}\text { Heart Attack(critical)(Highest Priority) } \\
\text { Heart Attack(mild) }\end{array}$ \\
\hline : & \\
\hline$:$ & \\
\hline : & \\
\hline $\mathrm{N}$ & Fever (Mild) (Lowest Priority) \\
\hline
\end{tabular}

vi. $\quad 0$ depicts the most critical condition followed by $1,2,3 \ldots \mathrm{N}$, where $\mathrm{N}$ depicts the least emergency.

vii. After making the necessary check up the nurse enters the relevant condition code in the GPS Enabled CMS Device.

viii. The Message body comprises of two sections : -

(i) The Location - This contains the GPS Coordinates and is automatically entered by the device.

(ii) Condition Code - An integer from 0 to $\mathrm{N}$ which depicts the condition of the patient.

ix. The Central Database collects all information regarding each and every Access Points. The Central Database processes the GPS Coordinates and inserts the value of the condition code for that particular vehicle across all the Access Points present en route to the hospital.

This way we can have the Access Point grant the Green Light for the Emergency Vehicle with highest priority (lowest condition code).

\section{REFERENCES}

[1] Monika Johri, Anurag Goel, Ashutish Kr. Tiwari "Dynamic Traffic Control Algorithm in Intelligent Transport System through Wireless Sensor Networks”, IJESR 2012

[2] "Intelligent Traffic Light Flow Control System Using Wireless Sensors Networks.” Khalil M.Yousef, Jamal N.Al - Karaki and Ali M.Shatnawi. JOURNAL OF INFORMATION SCIENCE AND ENGINEERING 26, 753-768 (2010)

[3] “Traffic Surveillance by Wireless Sensor Networks - Final report” Sing Yui Chen, Pravin Varaiya California Path Research Report UCB-ITS-PRR-2007-4

[4] "A Wireless Sensor Network for Traffic Surveillance”, Sing Yiu Cheung, Sinem Coleri, Ram Rajagopal,Pravin Varaiya

[5] "Wireless Sensor Networks - Wikipedia” http://en.wikipedia.org/wiki/Wireless_sensor_network

[6] “A Real Time Dynamic Traffic Control using Wireless Sensor Networks" CHEN Wenjie, CHEN Lifeng, CHEN Zhanglong, TU Shiliang Department of Computer Science and Engineering, Fudan University IEEE- 1530-2016/05 @ 2005

[7] "The Research of WSN-based Vehicle Information Detection Technology" Shen Ming Yu , Wang Wei. School of Computer and Information, Hefei University of Technology, Hefei 230009, China 2012 International Conference on Electronics, Information and Communication Engineering

[8] "A Distributed Algorithm for Multiple Intersections Adaptive Traffic Lights Control using a Wireless Sensor Networks" Sebastien Faye, Claude Chaudet, Isabelle Demuere - Institut Mines-Télécom CNRS LTCI UMR 5141

[9] "Comparison of Simulators for Wireless Sensor Networks" Martin Sehlik Master Thesis - Spring 2011 Faculty of Informatics Masaryk University

[10] “Time Complexity” http://en.wikipedia.org/wiki/Time_complexity

[11] "Merge Sort” http://bigocheatsheet.com/ 